\title{
Anthropisation Et Dynamique Des Paysages En Pays Agonlin Au Bénin
}

\author{
Damase Hintchémè Akpoyètè
}

Faculté des Sciences Humaines et Sociales (FASHS)

de l'Université d'Abomey-Calavi, Bénin

Laboratoire de Biogéographie et Expertise Environnementale (LABEE)

\section{Rodrigue C. Landeou}

Laboratoire de Biogéographie et Expertise Environnementale (LABEE)

Centre de Recherches Entomologiques de Cotonou (CREC), Bénin

\section{Vincent O. Orékan}

Faculté des Sciences Humaines et Sociales (FASHS) de l'Université d'Abomey-Calavi, Bénin, Laboratoire de Biogéographie et Expertise

Environnementale (LABEE)

\section{Doi: 10.19044/esj.2018.v14n36p571 URL:http://dx.doi.org/10.19044/esj.2018.v14n36p571}

\begin{abstract}
The temporal dynamics of landscapes in pays Agonlin zone of south Benin (West Africa) were studied through a diachronic analysis of three Landsat images (TM 1986, ETM 2000 et OLI TIR, 2015), supported by field visits. The results indicate that the forest cover was reduced from $35.46 \%$ in 1986 to $9.97 \%$ in 2016. This decrease of forest area went together with a loss of savannah area. On the other hand, agricultural areas increased up to $23.21 \%$ over the same period. Landscape pattern analysis by means of configuration metrics revealed a fragmentation of natural and seminatural vegetation by agricultural activities, leading to an aggregation of agricultural patches. As general trend, human activities and demographic growth have caused significant changes to the landscapes of the study region.
\end{abstract}

Keywords: Pays Agonlin, Landsat, Configuration metrics, Fragmentation, Anthropogenic effects

\section{Resume}

La dégradation des ressources forestières liée à des actions anthropiques expose le territoire du pays Agonlin à des désastres écologiques. Il est le principal lieu où se mènent anarchiquement les activités telles que l'agriculture, la transhumance, l'exploitation du bois d'œuvre et du bois de service, la recherche du bois de feu, la carbonisation et la chasse. La 
dynamique temporelle des paysages en pays Agonlin, situé dans le centre du Bénin en Afrique de l'Ouest, a été étudiée à partir de l'analyse diachronique de trois images satellitaires de type Landsat (TM 1986, ETM 2000 et OLI TIR, 2015), les données de terrain complétées par des missions de vérification sur le terrain. L'analyse de la structure spatiale du paysage a été également faite par des indices de configuration (aire moyenne, dimension fractale, dominance). Les résultats indiquent que les formations naturelles en 2016 couvraient $9,97 \%$ de la zone d'étude contre 35,46\% en 1986. Ce qui traduit la régression des formations naturelles. Par contre, les zones agricoles ont enregistré une progression de leur couverture de 23,21\% en 30 ans. L'analyse de la structure spatiale du paysage a montré une fragmentation des formations naturelles (forêts galeries, forêts claires savanes boisées) par les activités agricoles entraînant ainsi une fusion des taches agricoles. D'une façon générale, le milieu d'étude a subi une transformation importante liée essentiellement aux perturbations d'ordre anthropique et à la pression démographique.

Mots-clés: Pays Agonlin, Landsat, Anthropisation configuration, Fragmentation

\section{Introduction}

La dégradation des ressources forestières liée à des actions anthropiques expose le territoire du pays Agonlin à des désastres écologiques (Akpoyètè et $a l, 2017)$. Le pays Agonlin qui n'abrite aucune forêt classée de l'Etat (PAGEFCOM, 2012) est affecté à toutes sortes d'activités. Il est le principal lieu où se mènent anarchiquement les activités telles que l'agriculture, la transhumance, l'exploitation du bois d'œuvre et du bois de service, la recherche du bois de feu, la carbonisation et la chasse (Orékan, 2012 ; Wokou, 2009). Selon Ayikpon (2010), dans le pays Agonlin, « les défrichements anarchiques à des fins agricoles couplés au déboisement lié à la carbonisation et au commerce du bois d'œuvre débarrassent les terres de la couverture végétale ligneuse, notamment les mégaphanérophites, les mésophanérophites et une partie des microsphanérophites ; les paysans démunis, se reconvertissent par dizaine de centaines dans les activités de carbonisation, beaucoup de paysages végétaux subissent les feux de végétation, toutes les espèces d'arbres propices pour le bois d'œuvre sont prises d'assaut pour des utilisations diverses ».

Cette pression anthropique est due à la croissance démographique remarquable de sa population (Orékan, 2012). De 74352 en 1979 (RGPH1) la population passe à 95830 habitants en 1992, à 100917 en 2002 (RGPH3) ; et évaluée à 165689 en 2013 (RGPH4). En moins de 40 ans, la population du 
pays Agonlin a presque que doublé pendant que les ressources foncières et ligneuses s'amenuisent.

Aujourd'hui malgré l'élaboration du Schéma Directeur d'Aménagement Communal (SDAC) de chacune des trois Communes du pays Agonlin et du Schéma Territorial d'Aménagement et du Développement (STAD), les ressources forestières continuent de subir une forte pression anthropique (Akpoyètè, 2014 ; Ayikpon, 2010 ; Wokou, 2009). De même les nombreux projets (PAGEFCOM, PIFSAP, PAPDFGC, PBF II...) exécutés dans chacune des Communes du pays Agonlin, dans le souci de la préservation des ressources naturelles surtout végétales, peinent à donner des résultats concluants.

La présente étude vise à appliquer les techniques d'écologie du paysage à partir de trois images Landsat afin de caractériser la configuration spatiale du paysage par le calcul d'indices paysagers (McGarigal \& Marks, 1995). Ces derniers sont des indicateurs nécessaires pour mieux quantifier la fragmentation et l'anthropisation des éléments du paysage afin d'orienter rationnellement la politique agricole dans la zone. Pour atteindre cet objectif, il a été émis l'hypothèse selon laquelle, en pays Agonlin, le développement des activités agricoles couplé à l'exploitation forestière entraîne un changement de la structure spatiale du paysage.

\section{Cadre Matériel et méthodes d'étude Cadre d'étude}

Situe entre $6^{\circ} 8^{\prime}$ et $7^{\circ} 34^{\prime}$ latitude Nord d'une part, et $2^{\circ} 8^{\prime}$ et $2^{\circ} 33^{\prime}$ longitude Est d'autre part, le Pays Agonlin s'étend sur environ $68 \mathrm{~km} \mathrm{du}$ Sud au Nord et couvre une superficie de $1347 \mathrm{~km}^{2}$, soit $1,06 \%$ du territoire national (PAGEFCOM, 2012). Il est composé de trois Communes (Covè, Zagnanado et Ouinhi) qui sont subdivisées en 18 arrondissements) (figure 1). Le climat est de type subéquatorial avec deux saisons pluvieuses (mars-juillet et septembre novembre) et deux saisons sèches alternées (août-septembre et novembre-mars). La moyenne annuelle des hauteurs de pluie est $1200 \mathrm{~mm} / \mathrm{an}$. La température moyenne est de l'ordre de $27^{\circ} \mathrm{C}$, l'insolation avoisine 1800 $\mathrm{H} / \mathrm{an}$, alors que l'humidité relative varie d'un minimum de $78 \%$ en janvier/février à un maximum de $95 \%$ en septembre (ASECNA, 2016). La moyenne interannuelle de l'évapotranspiration (ETP) est de $280 \mathrm{~mm}$. Les différentes facettes pédologiques en pays Agonlin sont essentiellement de type ferralitique et hydromorphe (G. Wokou, 2009). Le pays Agonlin s'étend sur quatre bassins versants. Il présente un réseau hydrographique très dense et divisé en deux sous bassins (J. Houndagba, 1985, p.155). À l'échelle du Pays Agonlin existe encore de la végétation naturelle (forêt dense humide, forêt claire, forêt-galerie et savane boisée) (D. Akpoyètè, 2014, p.53). La population en pleine progression a atteint 165689 en 2013 (INSAE, RGPH, 2014). 
L'agriculture, la pêche, la chasse, l'exploitation forestière, la carbonisation sont autant d'activités exercées par les populations du milieu d'étude, principales causes de la fragmentation du paysage.

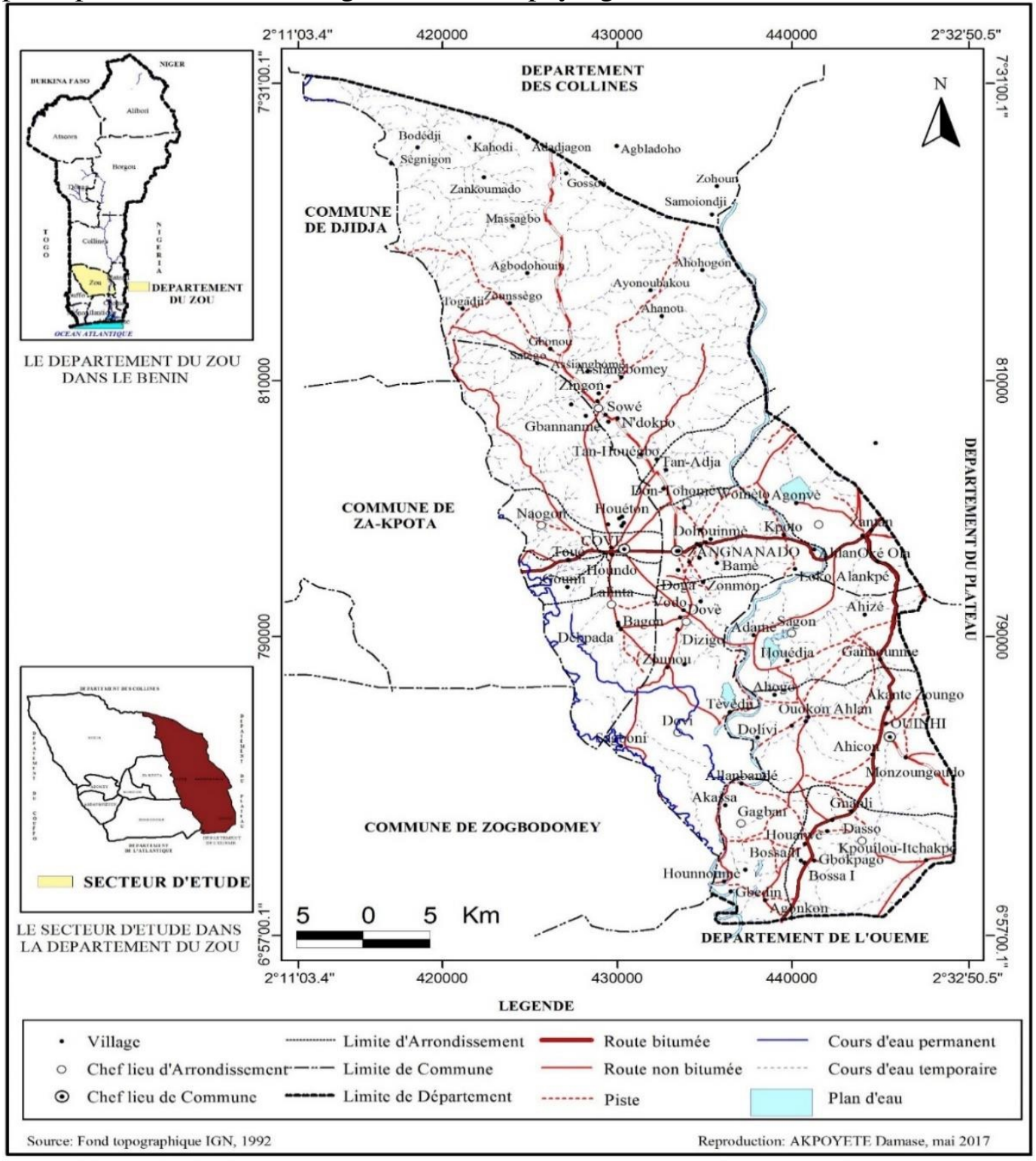

Figure 2: Localisation géographique du pays Agonlin

\section{Données satellitaires et auxiliaires}

Les différents résultats proviennent de l'exploitation de trois séries d'images satellitaires Thematic Mapper (TM) du 21/10/1986, Enhanced Thematic Mapper Plus (ETM) du 12/01/2000 et OLI TIRS 2015. Des cartes topographiques au 1:200.000 ont été utilisées comme cartes de base ainsi que 
les enquêtes socioénomiques de terrain et les documents de l'Archive Nationale de la République du Bénin.

\section{Méthode de traitement et d'analyse Prétraitement des images}

Les trois images ont fait l'objet de prétraitement afin de corriger les déformations géométriques et radiométriques des plates-formes et des capteurs spécifiques (Bonn et Rochon, 1992). La correction géométrique appliquée a consisté à ajuster certains points de l'image avec des zones invariantes au sol. La précision géométrique du calage entre les trois scènes est inférieure à 1 pixel, ce qui correspond au minimum requis pour une analyse des changements (Mas, 2000). Les méthodes de régressions linéaires fondées sur des invariants radiométriques (Song et al., 2001) ont été utilisées pour effectuer la correction radiométrique (Barima, 2009). Elles consistent à rendre l'image plus lisible et plus interprétable par rapport à l'image thématique.

Une composition colorée en fausses couleurs a ensuite été effectuée sur les images Landsat 8 corrigées à partir des bandes vert $(\mathrm{V})$, rouge $(\mathrm{R})$ et proche infrarouge (PIR). Ces canaux sont classiquement utilisés pour discriminer les classes de végétation. Mais, c'est surtout les canaux R et PIR qui permettent la plus grande différenciation des classes de végétation (Bonn et Rochon, 1992 ; Chatelain et al., 1996).

\section{Classification des images}

Sur la base des connaissances du terrain, il a été adopté la classification supervisée avec l'algorithme du « maximum de vraisemblance » pour identifier les classes d'occupation du sol en 1986, 2000 et 2016. Lors de l'utilisation de la méthode de classification supervisée, des échantillons homogènes et représentatifs de l'image des différents types de surfaces sont au préalable identifiés et interprétés à partir de la composition colorée. Celleci fait ressortir en rouge les formations arborées denses et encore feuillues que sont les forêts galeries, les forêts claires et les savanes boisées. Les savanes arborées et arbustives apparaissent en bleu vert, du fait de l'assèchement de la couverture herbacée en saison sèche. Elles réfléchissent assez bien dans les bandes spectrales visible et thermique, mais pas dans le proche infrarouge. Enfin, les sols nus et les terres cultivables après récolte réfléchissent fortement aussi bien le proche infrarouge que le visible et le thermique et ressortent en blanc.

Une classification supervisée commence donc par l'identification des classes d'information qui sont ensuite utilisées pour définir les classes spectrales qui les représentent (Mas, 2000). Après avoir délimité la zone d'étude, il a été utilisé l'algorithme du "maximum de vraisemblance » (maximum likehood) pour effectuer la classification. Cet algorithme utilise 
des zones échantillons pour déterminer les caractéristiques des classes d'objets, qui deviennent également des centres dans l'espace multispectral (Bonn et Rochon, 1992).

Les zones d'entrainement, représentatives de la diversité de chaque classe et de taille suffisante, doivent être bien dispersées sur l'ensemble de la zone d'étude. En tenant compte de ces critères et de la zone, pour chaque classe, eu égard au type d'image (Landsat) utilisé, nous avons réalisé plus de 25 zones d'entrainement par unité d'occupation

Enfin, un filtre «modal » avec une fenêtre de dimension $3 * 3$ a été applique à chaque image afin d'améliorer sa lisibilité.

\section{Vérification de la classification de la végétation}

La précision de la classification de la végétation a été vérifiée à partir des points identifiés sur les unités homogènes pour chacune des classes de végétation de l'image de 2016. Au niveau de chaque classe, les points de contrôle ont été localisés à l'aide du GPS et repartis autant que possible sur l'ensemble de la zone d'étude. Des visites de validation sur le terrain ont été effectuées pour confirmer et reclassifier l'interprétation de l'imagerie satellitaire. Au total, sur 225 points de contrôle échantillonnés, 221 se sont avérés correctement classés soit une proportion de $97 \%$.

\section{Mise en évidence et analyse de la dynamique}

La dynamique du paysage peut être décrite par des changements dans le temps des indices qui décrivent la structure et la composition du paysage (Schlaepfer, 2002) mais aussi par d'autres approches complémentaires telles que la matrice de transition et l'identification des processus de transformation spatiale (Schlaepfer, 2002; Barima, 2009).

La structure du paysage est caractérisée, pour chaque classe (j) d'occupation du sol, à partir du nombre de tache $\left(\mathrm{n}_{\mathrm{ij}}\right)$, de l'aire $\left(\mathrm{a}_{\mathrm{ij}}\right)$ et du périmètre $\left(\mathrm{p}_{\mathrm{ij}}\right)$ des taches de la classe et de quelques indices ont été calculés:

- l'indice de dominance $\mathrm{Dj}(\mathrm{a})$. Cet indice indique la proportion d'aire occupée par la tache dominante de la classe $\mathrm{j}\left(\mathrm{a}_{\mathrm{max}}, \mathrm{j}\right)$ dans l'aire totale de la classe (atj) (McGarigal et Marks, 1995).

Sa formule est : $\boldsymbol{D}_{\boldsymbol{j}}(\mathbf{a})=\frac{\boldsymbol{a}_{\max j}}{\boldsymbol{a}_{t j}} \mathbf{x} \mathbf{1 0 0}$ avec $a_{\max j}$ aire maximale de la classe $\mathrm{j}$ et $a_{t j}$ aire totale de la classe j.

Plus la valeur de la dominance est grande, moins la classe est fragmentée.

- la dimension fractale Dfj. Cet indice est considéré comme une mesure du degré d'anthropisation des taches dans une classe du paysage (Krummel et al., 1987). Elle utilise essentiellement le principe du ratio entre périmètre et surface, proposée par Mandelbrot (1994) dans le but de quantifier selon Iorgulescu et Schlaepfer (2000) la forme d'objets complexes. 
La formule de base est : $\boldsymbol{P}_{i j}=\boldsymbol{K} . \boldsymbol{a}_{i j} \boldsymbol{D f} \boldsymbol{f}_{j}$, avec Pij, la somme totale du contours de chacune des taches d'une classe.

Soit, en utilisant une transformation logarithmique $: \log p_{i j}=\log K+\left(\mathbf{D} f_{j} / 2\right) \cdot \log$

$\boldsymbol{a}_{i j}$

Dfj est la dimension fractale de la classe $\mathrm{j}$ et $\mathrm{k}$ une constante.

La dimension fractale de la classe $\mathrm{j}$ est donc obtenue à partir de la régression linéaire entre les aires (log aij) et les périmètres (log pij) de l'ensemble des taches de la classe. Le graphique log-log donne la pente de la régression qui est égale à $\mathrm{Dfj} / 2$. La valeur de la dimension fractale est donc le double de la valeur de la pente de la droite de régression obtenue. Elle varie de 1 à 2. Lorsque Dfj tend vers 2, les taches ont des formes complexes (milieu naturel moins anthropisé) ; lorsque Dfj tend vers l'unité, les taches ont des formes simples ou régulières (carré, rectangle ...) le plus souvent liées aux activités anthropiques (Krummel et al., 1987 ; Iorgulescu et Schlaepfer, 2000).

La matrice de transition utilisée permet de décrire de manière condensée, sous forme de matrice carrée, les changements et les affectations d'occupation du paysage au cours de l'intervalle de temps (Schlaepfer, 2002). Les cellules de la matrice contiennent la valeur d'une aire ayant passé d'une classe initiale $\mathrm{i}$ à une classe finale $\mathrm{j}$ pendant la période considérée. Les valeurs des colonnes représentent les proportions des aires occupées dans le paysage par chaque classe d'occupation du paysage au temps $\mathrm{j}$ et celles des lignes, au temps initial $\mathrm{i}$.

Le processus de transformation spatiale a été déterminé à l'aide d'un arbre de décision propose par Bogaert et al. (2004) pour une analyse complémentaire de la dynamique structurelle du paysage. Les données à introduire dans l'arbre de décision sont le nombre $\left(n_{j}\right)$ de taches, la surface $\left(a_{j}\right)$, le périmètre $\left(\mathrm{p}_{\mathrm{j}}\right)$ appartenant à la classe $\mathrm{j}$ aux temps initial et final de l'étude. Ces trois éléments sont considérés comme étant les éléments principaux de la description de la configuration paysagère (Giles et Trani, 1999). Le changement de chacun des trois éléments est utilisé pour aboutir à un processus représentatif de la dynamique observée. Les données relatives à ces trois éléments sont calculées pour l'image de 1986 ( $\mathrm{a}_{1986}, \mathrm{p}_{1986}$ et $\left.\mathrm{n}_{1986}\right)$ celle de 2000 (a2000, $\mathrm{p}_{2000}, \mathrm{n}_{2000}$ ) et celle de 2016 ( $\mathrm{a}_{2016}, \mathrm{p}_{2016}, \mathrm{n}_{2016}$ ). Selon le modèle, il faut d'abord analyser l'évolution du nombre $\left(\mathrm{n}_{\mathrm{j}}\right)$ de taches entre les deux dates (comparaison de $n_{1986}$ avec $\left.n_{2000}\right)$ et ( $n_{2000}$ avec $\left.n_{2016}\right)$.

Un premier niveau de hiérarchisation du processus de transformation spatiale peut être effectué. Par la suite, le changement de l'aire (comparaison de $\mathrm{a}_{1986}$ et de $\mathrm{a}_{2000}$ ) ou ( $\mathrm{a}_{2000}$ avec $\mathrm{a}_{2016}$ ) du type considéré mènera directement vers un des processus ou indirectement via l'évolution du périmètre (comparaison $\mathrm{p}_{1986}$ et $\mathrm{p}_{2000}$ ) ou ( $\mathrm{p}_{2000}$ avec $\mathrm{p}_{2016}$ ). C'est ainsi que dix processus ont pu être distingués : la création (apparition) $\left(\mathrm{n}_{2000}>\mathrm{n}_{1986}\right.$ et $\left.\mathrm{a}_{2000}>\mathrm{a}_{1986}\right)$, la dissection et la fragmentation $\left(\mathrm{n}_{2000}>\mathrm{n}_{1986}\right.$ et $\left.\mathrm{a}_{2000}<\mathrm{a}_{1986}\right)$; la distinction entre 
les processus de fragmentation et de dissection nécessite l'utilisation d'une valeur prédéfinie de diminution d'aire (t) (Bogaert et al., 2004) qui, comparée à tobs, dissocie ces deux processus, Avec : tobs $=a_{2000} / a_{1986}$

Le processus dominant est la fragmentation si tobs < t, et la dissection dans le cas contraire. Dans le cadre de notre étude, nous avons retenu $\mathrm{t}=0,5$ comme valeur seuil. Cette valeur seuil a été utilisée par plusieurs auteurs (Barima, 2009 ; Barima et al., 2009). Les autres processus sont l'agrégation $\left(\mathrm{n}_{2000}<\mathrm{n}_{1986}, \mathrm{a}_{2000}>\right.$ a1986) et la suppression $\left(\mathrm{n}_{2000}<\mathrm{n}_{1986}, \mathrm{a}_{2000}<\mathrm{a}_{1986}\right)$. Lorsque le nombre de taches pour les deux dates est identique $\mathrm{n}_{2000}=\mathrm{n}_{1986}$, il y a soit un agrandissement ( $\left.\mathrm{a}_{2000}>\mathrm{a}_{1986}\right)$, soit si $\mathrm{a}_{2000}<\mathrm{a}_{1986}$, le changement du périmètre (comparaison $\mathrm{p}_{2004}$ et $\mathrm{p}_{1985}$ ) conduit vers les processus de perforation $\left(\mathrm{p}_{2000}>\mathrm{p}_{1986}\right)$, de rétrécissement $\left(\mathrm{p}_{2000}<\mathrm{p}_{1986}\right)$, de déformation $\left(\mathrm{p}_{2000} 61 / 4 \mathrm{p}_{1986}\right)$ et de déplacement $\left(\mathrm{p}_{2000}=\mathrm{p}_{1986}\right)$.

\section{Résultats}

\section{Cartographie et classes de végétation}

Suite aux traitements numériques des données, neuf classes d'occupation du sol (figure 2 et figure 3 et figure 4) ont été définies en pays Agonlin: forêt galerie, forêt claire savane boisée, savane arborée arbustive, prairie marécageuse, plantation, culture et jachère sous palmier, mosaïque de culture et jachère, agglomération et les plans d'eau.

\section{Etat du paysage en 1986, 2000 et 2016}

L'analyse de la figure 3 et du tableau 1 indique que le paysage du pays Agonlin était constitué en 1986 de 3,14\% de forêt galerie, 32,32\% de forêt claire savane boisée, 34,30 \% de savane arborée arbustive, de 11,14\% de prairie marécageuse, de 7,81 de mosaïque de culture et jachère, de $8,92 \%$ de culture et jachère, de $0,6 \%$ des plantations, de 1,1 des points d'eau et de 0,5 $\%$ des agglomérations.

L'analyse de la figure 4 et du tableau 2 indique que le paysage du pays Agonlin était constitué en 2000 de 2,82 \% de forêt galerie, 14,91\% de forêt claire savane boisée, 28,79\% de savane arborée arbustive, de 10,05\% de prairie marécageuse, de 20,68 de mosaïque de culture et jachère, de 13,15\% de culture et jachère, de $3,96 \%$ des plantations, de $1,2 \%$ des points d'eau et de $1,1 \%$ des agglomérations.

En 2016, la composition du paysage révèle, selon la figure 4 et le tableau 3, 1,45 \% de forêt galerie, 8,52 \% de forêt claire savane boisée, 23,20 $\%$ de savane arborée arbustive, de $09,11 \%$ de prairie marécageuse, de 32,46 de mosaïque de culture et jachère, de 7,58 \% de culture et jachère, de 10,62 \% des plantations, de 1,18\% des points d'eau et de 2,67 \% des agglomérations. Mise en évidence des changements à l'échelle du paysage entre 1986 et 2000 
L'examen de la matrice de transition (tableau I) permet de mieux cerner les différents changements subis par la végétation de 1986 à 2000 :

\section{- $\quad$ Régression}

Les unités d'occupation qui ont régressé sont essentiellement les formations végétales naturelles :

$\checkmark \quad$ forêts galeries : De 1986 à 2000, la superficie des forêts galeries est passée de 4354 hectares à 3917 hectares. Il est noté que 194 hectares de savanes arborées arbustives, 1325 hectares de prairies marécageuses, 10 hectares de mosaïques de cultures et jachères et 100 hectares de cultures et jachères sous palmier ont été le résultat de conversion des forêts galeries.

$\checkmark \quad$ forêts claires savanes boisées : De 1986 à 2000, la superficie des forêts claires et des savanes boisées est passée de 44747 à 20651 hectares. Il est er noté que 8201 hectares de savanes arborées arbustives, 165 hectares de mosaïques de cultures et jachères, 4662 hectares de plantations et 6400 hectares de cultures et jachères sous palmier ont été le résultat de conversion des forêts claires savanes boisée.

$\checkmark \quad$ savanes arborées et arbustives : De 1986 à 2000, la superficie des savanes arborées et arbustives est passée de 47488 à 39861 hectares. Il est à noter que 14522 hectares de mosaïques de cultures et jachères, 462 hectares d'agglomérations et 2000 hectares de cultures et jachères sous palmier ont été le résultat de conversion des savanes arborées arbustives.

$\checkmark \quad$ prairies marécageuses : De 1986 à 2000, la superficie des prairies marécageuses est passée de 15435 à 13922 hectares. Il est noté que 62 hectares de savanes arborées arbustives, 148 hectares de mosaïques de cultures et jachères, 178 hectares de points d'eau et 600 hectares de cultures et jachères sous palmier ont été le résultat de conversion des prairies marécageuses.

\section{- Progression}

Les unités d'occupation qui ont connu de progrès sont :

$\checkmark \quad$ agglomération : le tableau I indique que les agglomérations passent de 703 à 1665 hectares. Il est noté que 143 hectares de mosaïque des cultures et jachères, 357 hectares de cultures et jachères sous palmiers et 462 hectares de savanes arborées arbustives ont été convertis en agglomérations ;

$\checkmark \quad$ mosaïque de cultures et jachères : De 1986 à 2000, la superficie de mosaïque des cultures et jachères a connu une progression rapide passant de 10824 hectares à 28635 hectares. Il est noté que cette progression est le résultat des conversions de 10 hectares de forêts galeries, 165 hectares de forêts claires et savanes boisées, 14522 hectares de savanes arborées arbustives, 3093 hectares de cultures et jachères sous palmier et 148 hectare de prairie marécageuse.

$\checkmark \quad$ Culture et jachère sous palmiers : de 1986 à 2000, la superficie culture et jachère sous palmiers a connu une progression passant de 1235 hectares à 18212 hectares. Il est noté que cette progression est le résultat des conversions 
de 100 hectares de forêts galeries, 6400 hectares de forêts claires et savanes boisées, 2000 hectares de savanes arborées arbustives, 26 hectares de mosaïque de cultures et jachères et 600 hectares de prairie marécageuse et 186 hectares de plantations.

$\checkmark \quad$ Plantation : de 1986 à 2000, la superficie des plantations a connu une progression passant de 900 hectares à 5488 hectares. Il est noté que cette progression est le résultat des conversions de 4662 hectares de forêts claires et savanes boisées, 833 hectares de savanes arborées arbustives et 2 hectares de cultures et jachères sous palmier.

Mise en évidence des changements à l'échelle du paysage entre 2000 et 2016

L'analyse de l'évolution du paysage à partir de la matrice de transition (Tableau II), permet de mieux cerner les différentes transformations subies par la végétation de 2000 à 2016 :

\section{- régression}

Les unités d'occupation qui ont régressé sont :

$\checkmark \quad$ forêts galeries : De 2000 à 2016, la superficie des forêts galeries est passée de 3917 à 2015 hectares. Il est noté que 964 hectares de mosaïques de cultures et jachères, 418 hectares de plantations et 520 hectares de cultures et jachères sous palmier ont été le résultat de conversion des forêts galeries ;

$\checkmark \quad$ forêts claires et savanes boisées : De 2000 à 2016, la superficie des forêts claires et savanes boisées est passée de 20651 à 11803 hectares. Il est noté que 430 savanes arborées arbustives, 5536 de mosaïques de cultures et jachères, 201 hectares de cultures et jachères sous palmiers, 1929 hectares de plantations et 718 hectares d'agglomérations ont été le résultat de conversion des forêts claires savanes boisées ;

$\checkmark \quad$ savanes arborées et arbustives : De 2000 à 2016, la superficie des savanes arborées et arbustives est passée de 39861 à 32121 hectares. Il est noté que 6029 de mosaïques de cultures et jachères, 1941 hectares de plantations et 200 hectares d'agglomérations ont été le résultat de conversion des savanes arborées arbustives ;

$\checkmark \quad$ prairies marécageuses : De 2000 à 2016, la superficie des prairies marécageuses est passée de 13922 à 12616 hectares. Il est noté que 678 hectares de mosaïques de cultures et jachères, 731 hectares de plantations, 101 hectares de points d'eau et 4 hectares d'agglomérations ont été le résultat de conversion des prairies marécageuses ;

$\checkmark \quad$ Champs et jachères sous palmier : De 2000 à 2016, la superficie des champs et jachères sous palmier est passée de 18212 à 10494 hectares.

\section{- progression :}

Les unités d'occupation qui ont connu de progrès sont : 
$\checkmark \quad$ agglomération : De 2000 à 2016, la superficie des agglomérations est passée de 1655 à 3709 hectares. L'examen de la matrice de transition montre que 846 hectares de mosaïque de cultures et jachères, 718 hectares de forêts claires savanes boisées, 286 hectare de champ et jachère sous palmiers, 200 hectares de savanes arborées arbustives et 4 hectare de prairie marécageuse ont été convertis en agglomérations ;

Mosaïque de cultures et jachères : De 2000 à 2016, la superficie de mosaïque des cultures et jachères a connu une progression passant de 28635 à 44947 hectares. Il est noté que cette progression est le résultat des conversions de 964 hectares de forêts galeries, 5536 hectares de forêts claires et savanes boisées, 6029 hectares de savanes arborées arbustives, 678 hectares de prairies marécageuses, 5063 hectares de cultures et jachères sous palmier ;

$\checkmark \quad$ Plantation : De 2000 à 2016, la superficie des plantations est passée de 5488 à 14709 hectares. Il est noté que cette progression est le résultat des conversions de 418 hectares de forêts galeries, 1929 hectares de forêts claires et savanes boisées, 1941 hectares de savanes arborées arbustives, 731 hectares de prairies marécageuses, 3090 hectares de cultures et jachères sous palmier et 2318 hectares de mosaïques de cultures et jachères.

Tableau IV : Matrice de transition entre 1986 et 2000

\begin{tabular}{|l|l|l|l|l|l|l|l|l|l|l|}
\hline & $F G$ & $F C S B$ & $S A S A$ & $P M$ & $M C J$ & $C J P$ & $P L$ & $P E$ & $A G$ & TOTAL \\
\hline$F G$ & 39,17 & 0 & 0 & 1,325 & 2,04 & 1 & 0 & 0 & 0 & 43,545 \\
\hline$F C S B$ & 0 & 206,51 & 82,01 & 0 & 1,65 & 64 & 46,62 & 0 & 0 & 447,476 \\
\hline$S A S A$ & 0 & 0 & 305,04 & 0 & 145,22 & 20 & 0 & 0 & 4,62 & 474,88 \\
\hline$P M$ & 0 & 0 & 9,62 & 137,25 & 1,48 & 6 & 0 & 1,78 & & 154,35 \\
\hline$M C J$ & 0 & 0 & 0 & 0 & 106,55 & 0,26 & 0 & 0 & 1,43 & 108,24 \\
\hline$C J P$ & 0 & 0 & 0 & 0 & 30,93 & 89,00 & 0,02 & 0 & 3,57 & 123,5 \\
\hline$P L$ & 0 & 0 & 0 & 0 & 0 & 1,86 & 7,13 & 0 & 0 & 9 \\
\hline$P E$ & 0 & 0 & 0 & 0,64 & 0 & 0 & 0 & 15,65 & 0 & 16,297 \\
\hline$A G$ & 0 & 0 & 0 & 0 & 0 & 0 & 0 & 0 & 7,03 & 7,03 \\
\hline TOTAL & 39,17 & 206,51 & 396,67 & 139,22 & 288,29 & 182,12 & 54,88 & 17,43 & 16,65 & \\
\hline
\end{tabular}

Légende $:$ FG $=$ Forêt Galerie $;$ FCSB $=$ Forêt Claire et Savane Boisée ; SASA = Savane Arborée et Arbustive $; \mathrm{PM}=$ Prairie Marécageuse $; \mathrm{PL}=$ Plantation $; \mathrm{MCJ}=$ Mosaïque de Culture et Jachère ; CJP $=$ Culture et Jachère sous Palmier ; $\mathrm{PE}=$ Point d'Eau ; $\mathrm{AG}=$ Agglomération.

Source: interprétation Landsat TM, 1986 et Landsat ETM, 2000 
Tableau V: Matrice de transition entre 2000 et 2016

\begin{tabular}{|l|l|l|l|l|l|l|l|l|l|l|}
\hline & $F G$ & $F C S B$ & $S A S A$ & $P M$ & $M C J$ & $C J P$ & $P L$ & $P E$ & $A G$ & $T O T A L$ \\
\hline$F G$ & 20,15 & 0 & 0 & 0 & 9,64 & 5,20 & 4,18 & 0 & 0 & 39,17 \\
\hline$F C S B$ & 0 & 118,03 & 4,3 & 0 & 55,36 & 2,01 & 19,29 & 0 & 7,18 & 206,51 \\
\hline$S A S A$ & 0 & 0 & 316,91 & 0 & 60,29 & 0 & 19,41 & 0 & 2 & 398,61 \\
\hline$P M$ & 0 & 0 & 0 & 124,08 & 6,78 & 0 & 7,31 & 1,01 & 0,04 & 139,22 \\
\hline$M C J$ & 0 & 0 & 0 & 0 & 254,71 & 0 & 23,18 & 0 & 8,46 & 286,35 \\
\hline$C J P$ & 0 & 0 & 0 & 0 & 50,63 & 97,73 & 30,90 & 0 & 2,86 & 182,12 \\
\hline$P L$ & 0 & 0 & 0 & 0 & 12,06 & 0 & 42,82 & 0 & 0 & 54,88 \\
\hline$P E$ & 0 & 0 & 0 & 2,08 & 0 & 0 & 0 & 15,35 & 0 & 17,43 \\
\hline AG & 0 & 0 & 0 & 0 & 0 & 0 & 0 & 0 & 16,55 & 16,55 \\
\hline TOTAL & 20,15 & 118,03 & 321,21 & 126,16 & 449,47 & 104,94 & 147,09 & 16,36 & 37,09 & \\
\hline
\end{tabular}

Légende : FG = Forêt Galerie $;$ FCSB $=$ Forêt Claire et Savane Boisée $;$ SASA = Savane Arborée et Arbustive $; \mathrm{PM}=$ Prairie Marécageuse $\mathrm{PL}=$ Plantation $; \mathrm{MCJ}=$ Mosaïque de

Culture et Jachère ; CJP $=$ Culture et Jachère sous Palmier ; $\mathrm{PE}=$ Point d'Eau ; $\mathrm{AG}=$ Agglomération.

Source: Landsat ETM 2000 et Landsat OLI TIR 2015 plus travaux de terrain 


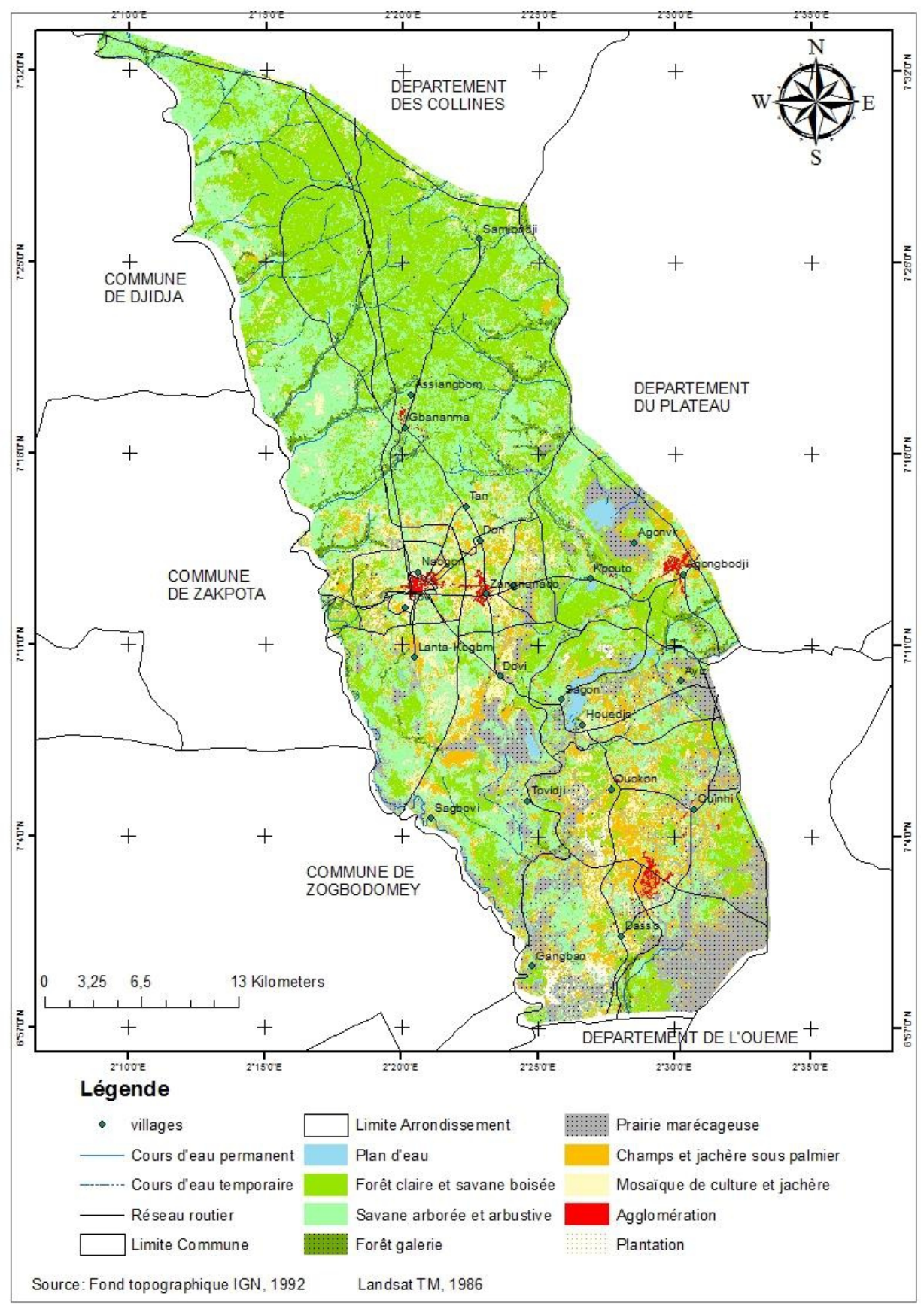

Figure 3: Etat du paysage en pays Agonlin en 1986 


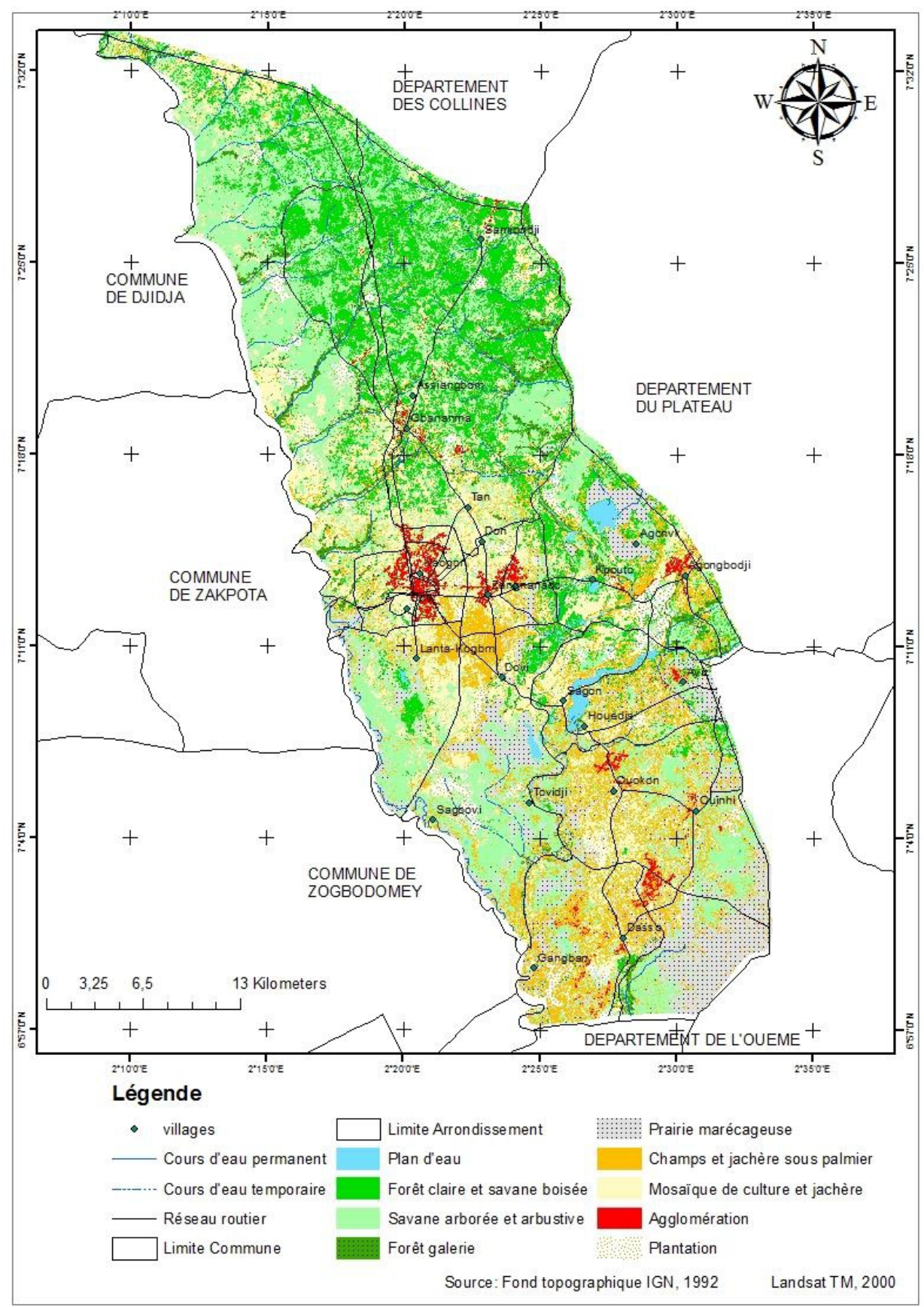

Figure 4 : Etat du paysage en pays Agonlin en 2000 


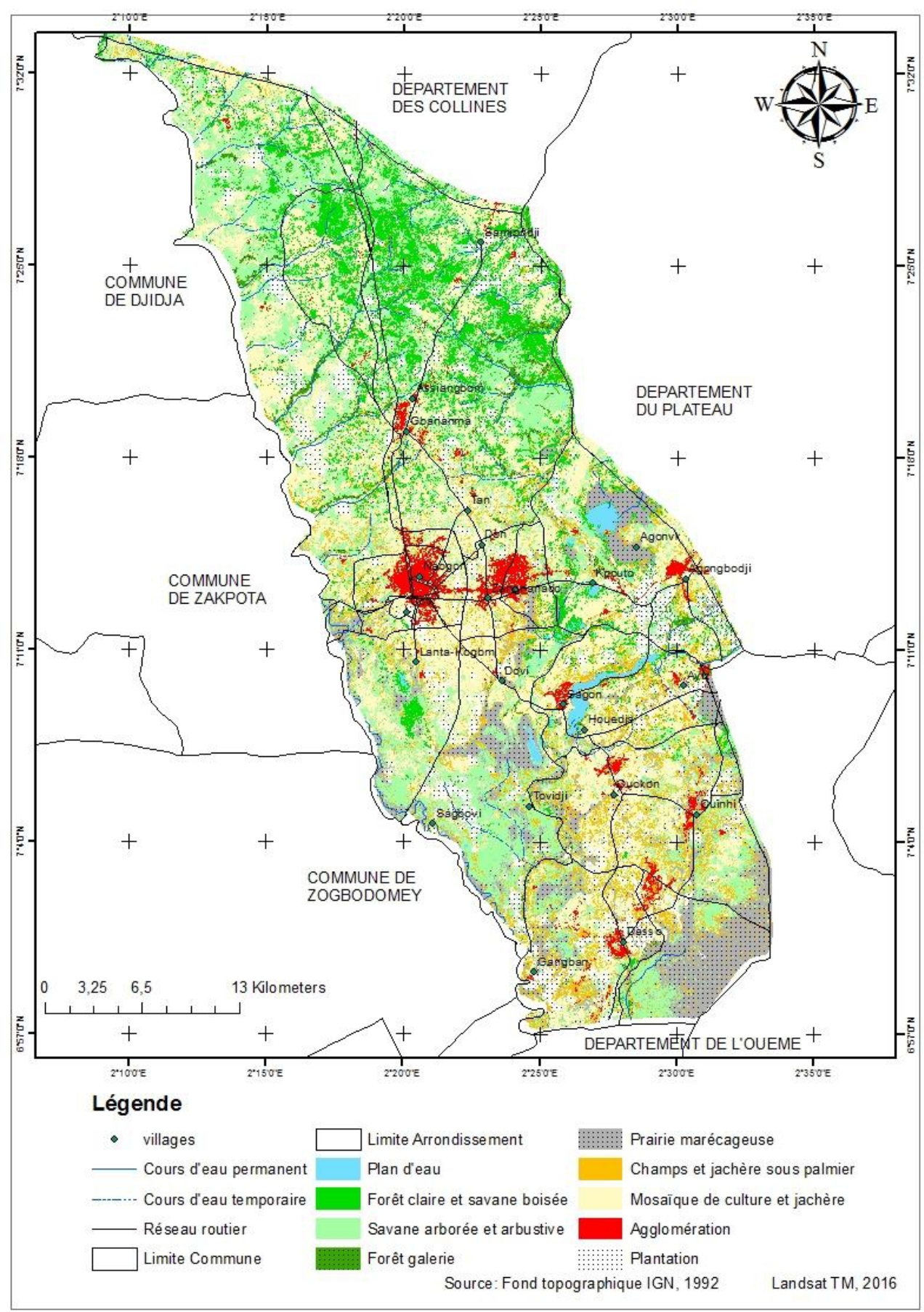

Figure 5 : Etat du paysage en pays Agonlin en 2016. 
Tableau VI: Nombre de tache, aire, aire moyenne, périmètre, dominance et forme des unités d'occupation en 1986 et 2000

\begin{tabular}{|c|c|c|c|c|c|c|c|c|c|c|}
\hline \multirow{2}{*}{$\begin{array}{l}\text { Unités } \\
\text { d'occupation }\end{array}$} & \multicolumn{2}{|c|}{ Aire total $\left(\mathrm{Km}^{2}\right)$} & \multicolumn{2}{|c|}{ périmètre $(\mathrm{Km})$} & \multicolumn{2}{|c|}{ nombre de taches } & \multicolumn{2}{|c|}{ Dominance } & \multicolumn{2}{|c|}{ Dimension fractale } \\
\hline & 1986 & 2000 & 1986 & 2000 & 1986 & 2000 & 1986 & 2000 & 1986 & 2000 \\
\hline $\mathrm{FG}$ & 43,5456 & 39,1716 & 58716 & 54108 & 275 & 275 & 0,1522 & 0,1498 & 1,033 & 1,0007 \\
\hline FCSB & 447,4764 & 206,5176 & 281916 & 203292 & 505 & 642 & 0,80729 & 0,7176 & 1,0366 & 1,004 \\
\hline SASA & 431,5032 & 398,6172 & 384444 & 320364 & 922 & 870 & 0,81159 & 0,1397 & 1,0339 & 1,0111 \\
\hline $\mathrm{PM}$ & 154,3536 & 139,2228 & 111312 & 102852 & 395 & 416 & 0,32256 & 0,32256 & 1,0279 & 1,0266 \\
\hline $\mathrm{PE}$ & 16,2972 & 17,4312 & 16164 & 17712 & 86 & 94 & 0,4059 & 0,4059 & 1,0291 & 1,0286 \\
\hline CJP & 123,5088 & 182,1204 & 156708 & 207360 & 708 & 758 & 0,11791 & 0,28897 & 1,0317 & 1,0257 \\
\hline MCJ & 108,2484 & 286,3512 & 151164 & 298188 & 806 & 1065 & 0,10196 & 0,69585 & 1,0256 & 1,0277 \\
\hline PL & 9,0072 & 54,8856 & 8316 & 39636 & 19 & 61 & 0,2392 & 0,6765 & 1,0525 & 1,0712 \\
\hline AGG & 7,0308 & 16,6536 & 7668 & 21852 & 34 & 113 & 0,1426 & 0,1715 & 1,029 & 1,0285 \\
\hline Total général & 1340,9712 & 1340,9712 & 1176408 & 1265364 & 3750 & 4294 & $\mathbf{3 2 , 5 5 3}$ & 33,0505 & 9,2993 & 9,2985 \\
\hline
\end{tabular}

Source : interprétation des images Landsat TM année, 1986 et Landsat MSS, année 2000

Tableau IV: Nombre de tache, aire, aire moyenne, périmètre, dominance et forme des unités d'occupation en 2000 et 2016

\begin{tabular}{|c|c|c|c|c|c|c|c|c|c|c|}
\hline \multirow{2}{*}{$\begin{array}{l}\text { Unités } \\
\text { d'occupation }\end{array}$} & \multicolumn{2}{|c|}{ Aire total $\left(\mathrm{Km}^{2}\right)$} & \multicolumn{2}{|c|}{ périmètre $(\mathrm{Km})$} & \multicolumn{2}{|c|}{ nombre de taches } & \multicolumn{2}{|c|}{ Dominance } & \multicolumn{2}{|c|}{ Dimension fractale } \\
\hline & 2000 & 2016 & 2000 & 2016 & 2000 & 2016 & 2000 & 2016 & 2000 & 2016 \\
\hline FG & 39,1716 & 20,1528 & 541080 & 318600 & 275 & 221 & 0,1498 & 0,0773 & 1,0007 & 1,0005 \\
\hline FCSB & 206,5176 & 118,0332 & 2032920 & 1442160 & 642 & 753 & 0,7176 & 0,21721 & 1,0004 & 1,00 \\
\hline SASA & 398,6172 & 321,2136 & 3203640 & 3095640 & 870 & 1041 & 0,1397 & 0,0536 & 1,0111 & 1,0326 \\
\hline PM & 139,2228 & 126,1656 & 1028520 & 898560 & 416 & 373 & 0,32256 & 0,317 & 1,0266 & 1,0265 \\
\hline $\mathrm{PE}$ & 17,4312 & 16,362 & 177120 & 159120 & 94 & 86 & 0,4059 & 0,4059 & 1,0286 & 1,0263 \\
\hline CJP & 182,1204 & 104,9436 & 2073600 & 1593360 & 758 & 984 & 0,28897 & 0,2368 & 1,0257 & 1,0256 \\
\hline MCJ & 286,3512 & 449,9712 & 2981880 & 3563280 & 1065 & 713 & 0,69585 & 0,92611 & 1,0277 & 1,0283 \\
\hline PL & 54,8856 & 147,096 & 396360 & 1254600 & 61 & 294 & 0,6765 & 0,9085 & 1,0712 & 1,0504 \\
\hline $\mathrm{AGG}$ & 16,6536 & 37,0332 & 218520 & 348120 & 113 & 128 & 0,1715 & 0,575 & 1,0285 & 1,0297 \\
\hline Total général & 1340,9712 & 1340,9712 & 12653640 & 12673440 & 4294 & 4593 & 33,0505 & 21,9435 & 9,2985 & 9,2665 \\
\hline
\end{tabular}

Source : interprétation des images Landsat MSS année, 2000 et Landsat TM, année 2016 
Le tableau III présente le nombre de tache, l'aire totale, le périmètre, la dominance et la dimension fractale des unités d'occupation en 1986 et 2000.

En 1986, les forêts galeries occupent une superficie de $43,5456 \mathrm{Km}^{2}$ avec comme nombre de tache 275 et comme périmètre $58716 \mathrm{Km}$. La dominance et la dimension fractale sont respectivement 0,1522 et 1,033. En 2000 cette même unité occupe une superficie de $39,1716 \mathrm{Km}^{2}$ avec comme nombre de tache 275 et comme périmètre $541080 \mathrm{Km}$. La dominance et la dimension fractale sont respectivement 0,1498 et de 1,0007.

En 1986, les forêts claires savanes boisées occupent une superficie de $447,4764 \mathrm{Km}^{2}$ avec comme nombre de 505 et comme périmètre $281916 \mathrm{Km}$. La dominance et la dimension fractale sont respectivement 0,80729 et de 1,0366. En 2000 cette même unité occupe une superficie de $206,5176 \mathrm{Km}^{2}$ avec comme nombre de tache 753 et comme périmètre $2032920 \mathrm{Km}$. La dominance et la dimension fractale sont respectivement de 0,7176 et de 1,0004 .

En 1986, les savanes arborées arbustives occupent une superficie de $431,5032 \mathrm{Km}^{2}$ avec comme nombre de tache 922 et comme périmètre 384444 $\mathrm{Km}$. La dominance et la dimension fractale sont respectivement 0,81159 et de 1,0339. En 2000 cette même unité occupe une superficie de $398,6172 \mathrm{Km}^{2}$ avec comme nombre de tache 870 et comme périmètre $3203640 \mathrm{Km}$. La dominance et la dimension fractale sont respectivement de 0,1397et de 1,0111 .

Entre 1986 et 2000, les formations naturelles que sont les forêts galeries, les forêts claires savanes boisées et les savanes arborées arbustives, ont donc connu diverses transformations dues à une anthropisation poussée comme l'indique la diminution des dimensions fractales qui tendent toutes vers 1 et les dominances qui ont chuté à tous les niveaux. Les forêts galeries ont connu une diminution de l'aire totale et de la dominance sans changement du nombre de tache pendant la période. Ce phénomène est qualifié du rétrécissement. Les forêts claires savanes boisées ont connu une augmentation du nombre de tache, une diminution de l'aire totale avec tobs < t. C'est le phénomène de fragmentation. Les savanes arborées arbustives ont connu une diminution du nombre de tache et de l'aire totale. C'est le phénomène de suppression.

Les paysages culturaux et bâtis ont confirmé cette tendance. Les mosaïques de culture et jachères, les cultures et jachères sous palmiers et les agglomérations ont connu une augmentation de leurs nombres de tache et de leurs superficies totales; ce qui indique le phénomène de création. La diminution de la dominance indique le caractère de subsistance de cette agriculture et le caractère rural des villages nouveaux.

Le tableau IV présente le nombre de tache, l'aire totale, le périmètre et la dominance des unités d'occupation en 2000 et 2016. L'examen de ce tableau 
permet de retenir quatre transformations majeures pendant la période 20002016.

En 2016, les forêts galeries occupent une superficie de $20,1528 \mathrm{Km}^{2}$ avec comme nombre de tache 221 et comme périmètre $318600 \mathrm{Km}$. La dominance et la dimension fractale sont respectivement 0,0773 et 1,0005.

En 2016, les forêts claires savanes boisées occupent une superficie de $206,5176 \mathrm{Km}^{2}$ avec comme nombre de 753 et comme périmètre $1442160 \mathrm{Km}$. La dominance et la dimension fractale sont respectivement 0,21721et de 1,00.

En 2016 les savanes arborées arbustives occupent une superficie de $321,2136 \mathrm{Km}^{2}$ avec comme nombre de tache 1041 et comme périmètre $3095640 \mathrm{Km}$. La dominance et la dimension fractale sont respectivement 0,0536 et de 1,0326 .

Pendant cette période l'anthropisation a été accélérée au niveau des formations naturelles comme l'indique la diminution des dimensions fractales qui tendent toutes vers 1 et des dominances qui ont chuté à tous les niveaux. Plusieurs taches de forêt galerie ont été supprimées, ce qui indique la diminution du nombre de tache et de l'aire totale. Les autres formations naturelles que sont les forêts claires savanes boisées, et les savanes arborées arbustive ont connu la dissection justifiée par la diminution de l'aire totale et l'augmentation du nombre de taches.

Les paysages culturaux et bâtis ont confirmé de nouveau cette tendance. Les mosaïques de culture et jachères et les agglomérations ont connu une augmentation de leurs nombres de tache et de leurs superficies totales ; ce qui indique le phénomène de création. La diminution de la dominance indique le caractère de subsistance de cette agriculture et le caractère rural des villages nouveaux.

\section{Discussion}

De 1986 à 2000, la superficie des forêts galeries est passée de 4354 hectares à 3917 hectares ; la superficie des forêts claires et des savanes boisées est passée de 44747 à 20651 hectares et la superficie des savanes arborées et arbustives est passée de 47488 à 39861 hectares. Par contre au cours de la même période, les agglomérations passent de 703 à 1665 hectares; la superficie de mosaïque des cultures et jachères passent de 10824 hectares à 28635 hectares et la superficie culture et jachère sous palmiers a connu une progression passant de 1235 hectares à 18212 hectares.

De 2000 à 2016, la superficie des forêts galeries est passée de 3917 à 2015 hectares ; la superficie des forêts claires et savanes boisées est passée de 20651 à 11803 hectares et la superficie des savanes arborées et arbustives est passée de 39861 à 32121 hectares. Au cours de la même période, la superficie des agglomérations est passée de 1655 à 3709 hectares et la superficie de 
mosaïque des cultures et jachères a connu une progression passant de 28635 à 44947 hectares.

Les résultats de cette étude ont montré que la fragmentation du paysage entraine la régression du paysage à couverture végétale et la progression du paysage cultural et habité. La dynamique du couvert végétal est un sujet qui a été abordé par plusieurs auteurs dans plusieurs régions du Bénin à l'instar de : Plagbéto (2014) dans le bassin inférieur de l'Ouémé Bonou, Ali (2012) dans la basse vallée de l'Ouémé dans le Bénin méridional, Arouna (2012) dans la Commune de Djidja au centre du Bénin, Sounon Bouko (2011) dans la Commune de Tchaourou au nord Bénin, Toko Mouhamadou (2014) dans les Monts Kouffé et de sa périphérie dans le nord Bénin. Tous ces auteurs sont unanimes sur le fait que les formations naturelles sont en plein recul au profit des cultures et jachères.

Ces résultats obtenus reflètent la réalité de la tendance de la dynamique du paysage au Bénin, où le passage d'une économie d'autosubsistance à une économie de marché, avec comme moteur la monoculture, est à la base des changements sociaux qui se répercutent aussi sur le paysage. Le constat le plus remarquable dans l'évolution de l'occupation du sol est l'évolution grandissante de l'emprise agricole qui s'accompagne d'une fragmentation (Bogaert \& Hong, 2003) et d'une homogénéisation des paysages via l'agrandissement des taches agricoles (Sabatier et al., 2010). Or, selon Bogaert et Mahamane (2005), l'anthropisation dans un milieu naturel est généralement caractérisée par la fragmentation, un changement dans la géométrie des taches et une évolution des classes anthropisées au détriment des classes naturelles d'occupation du sol. C'est ce qui confirme d'ailleurs les différentes transformations du couvert végétal entre 1986 et 2016 en pays Agonlin que sont la fragmentation, la dissection, le rétrécissement et la suppression du couvert végétal naturel suite à l'intervention humaine. Ces résultats sont similaires à Toko Mouhamadou (2014), Soumagbo (2010), Mama et al. (2013), Diallo et al. (2011).

\section{Conclusion}

Cette étude nous a permis, grâce aux méthodes de l'écologie du paysage associées à la télédétection et au Système d'Information Géographique, de quantifier la dégradation du paysage en pays Agonlin au centre du Bénin entre 1986, 2000 et 2016. Cette partie du Bénin dont la matrice paysagère était jadis dominée par les forêts et les savanes est aujourd'hui de plus en plus anthropisée.

L'accroissement de la population et les actions anthropiques accentuent les phénomènes de dégradation. L'étude a montré que l'occupation progressive du sol par des cultures est l'une des causes essentielles de la modification de la végétation. Elle a également mis en exergue les modes 
actuels d'utilisation des ressources végétales qui n'obéissent pas à des normes de durabilité. Cela constitue une inquiétude pour la conservation de l'écosystème en pays Agonlin. Pour contribuer à conserver l'écosystème et sa biodiversité, une sensibilisation des populations s'impose.

Il serait nécessaire d'envisager l'augmentation de la productivité par l'amélioration du système agraire archaïque au profit d'un système agrosylvopastoral (association cultures et légumineuses ligneuses, synergie agriculture- élevage), d'un procédé de culture intensive (plus économique du point de vue de la superficie) ou encore de rotation des cultures pour améliorer la fertilité des sols. La récupération des terres dégradées par la vulgarisation des techniques de défense et de restauration des sols ou par le reboisement des essences forestières locales utiles pour les populations s'avère également indispensable.

Enfin, la vulgarisation du nouveau code domanial voté en 2013 et corrigé en 2017 au Bénin pourrait être envisagée dans le cadre de la gestion de l'espace.

\section{References:}

1. Akpoyètè H.D., Landeou C. R., Aïtchéou A. et Orékan O. V., 2017. Impacts négatifs de la fragmentation du paysage sur les ressources en pays Agonlin. MIRD. Rech.Sci. Univ. d'Abomey-Calavi (Bénin), 8 (12) :254-267.

2. Akpoyètè H.D., 2014. Etat de fragmentation du paysage dans la Commune de Zagnanado: situation actuelle et perspectives. Mémoire de DESS en Géosciences de l'environnement et développement durable. CIFRED, Université d'Abomey-Calavi, 81p.

3. Ali M. F. K. R., 2012. Déterminants écologiques, anthropologiques et socio-économiques pour la conservation et la gestion durable des forêts sacrées et communautaires de la basse vallée de l'Ouémé dans le Bénin méridional. Thèse de doctorat, FLASH, Université d'Abomey-Calavi, $210 \mathrm{p}$.

4. Arouna O., 2012. Cartographie et modélisation prédictive des changements spatio-temporels de la végétation dans la commune de Djidja au Bénin : implication pour l'aménagement du territoire. Thèse de Doctorat, UAC/FLASH, 162 p.

5. Arouna O., 2002. L'exploitation des ressources biologiques et la dynamique de la forêt classée de l'Alibori supérieur (secteur de l'arrondissement de Bagou). Mémoire de maîtrise, UAC/FLASH/DGAT, 115 p.

6. Arouna O., Sinsin B. \& Houndagba C.J., 2002. L'exploitation des ressources biologiques et la dynamique de la forêt classée de l'Alibori supérieur au Bénin. Université d'Abomey- Calavi, 117 p. 
7. Barima YS., 2009. Dynamique, fragmentation et diversité végétale des paysages forestiers en milieu de transition foret-savane dans le département de Tanda (Cote d'ivoire). Thèse de doctorat, université libre de Bruxelles, 2009.

8. Barima YSS., Barbier N., Bamba I., Traore D., Lejoly J., Bogaert J., 2009. Dynamique paysagère en milieu de transition foret -savane ivoirienne. Bois et Forêts des Tropiques 299 : 15-25.

9. Bogaert J., Barima Y.S.S., Ji J., Jiang H., Bamba I., Iyongo W.M.L., Mama A., Nyssen E., Dahdouh-Guebas F. \& Koedam N., 2011. A methodological framework to quantify anthropogenic effects on landscape pattern. In: Landscape ecology in Asian cultures (eds S.-K. Hong, J. Wu, J.-E. Kim \& N. Nakagoshi), Springer, Verlag, New York, pp. 141-167.

10. Bogaert J. \& Mahamane A., 2005. Ecologie du paysage: cibler la configuration et l'échelle spatiale. Annales des Sciences Agronomiques du Bénin, 7, 39-68.

11. Bogaert J., Ceulemans, R., Salvador-Van D., 2004. Decision tree algorithm for detection of spatial processes in landscape transformation. Environmental Management, 33 : 62-73.

12. Bogaert J. \& Hong S.K., 2003. Landscape ecology: monitoring landscape dynamics using spatial pattern metrics. In: Ecological issues in a changing world, (eds. Hong, S.K.; Lee, J.A.; Ihm B.S.; Farina A.; Son Y.; Kim E.S. \& Choe J.C), Kluwer Academic Publishers, Dordrecht, pp. 109-131.

13. Bonn F. \& Rochon G., 1992, Précis de télédétection. Principes et méthodes. Sainte-Foy (Québec): Presses de l'Université du Québec, $485 \mathrm{p}$.

14. Chazdon R.L., Harvey C.A., Griffith D.M., Ferguson B. G., MartinezRamos M., Morales H., Nigh R., Soto-Pinto L., Breugel M., Philpot S. M., 2009. Beyong reserves: a research agenda for conserving biodiversity in human modified tropical landscapes. Biotropica, 41 (2):142-153.

15. Collinge S.K., 1998. Spatial arrangement of habitat patches and corridors: clues from ecological field experiments. Landscape and Urban Planning, 42, 157-168.

16. Corgne S., 2004. Modélisation prédictive de l'occupation des sols en contexte agricole intensif: application à la couverture hivernale des sols en Bretagne. Thèse de Doctorat, Haute Bretagne, France, 230 p.

17. Djego J. G. M., 2006. Phytosociologie de la végétation de sous-bois et impact écologique des plantations forestières sur la diversité floristique au Sud et au Centre du Bénin. Thèse de Doctorat, Université d'Abomey-Calavi, 388 p. 
18. Djogbénou C.P., 2010. Analyse multicritère des Plans d'Aménagement et de Gestion participatifs des forêts classées au Bénin : développement d'un modèle durable. Thèse de Doctorat, Université d'Abomey-Calavi, $226 \mathrm{p}$.

19. Giles RHJ, Trani MK, 1999. Hey elements of landscape pattern measures. Environment Management 23: 477-81.

20. Grenan P. \& Joiris D., 2000. Usages de l'espace et enjeux territoriaux en forêt tropicale. In: Bahuchet S. \& De Maret P. Editions. Avenir des peuples des forêts tropicales, 2, Approche thématique. Bruxelles: APFT, ULB, p. 107-134.

21. Hockings M., Stolton S., Leverington, F., Dudley N. et Courrau J., 2008. Évaluation de l'efficacité : Un cadre pour l'évaluation de l'efficacité de la gestion des aires protégées. UICN, 2ème édition, Gland, Suisse, $105 \mathrm{p}$.

22. Houndagba C. J., 1985. Analyse typologique des paysages d'AbomeyZagnanado. Exploitation d'un système de programme pli. Neptune .Thèse de doctorat de 3è cycle ULP. Strasbourg.- France, 286p.

23. INSAE., 2013. Recensement Général de la Population et de l'Habitation (RGPH4).

24. Iorgulescu K, Schlaepfer R, 2000. Paysage en tant qu'Eco complexe : définition, types, caractéristiques, fonctionnement et fonctions. Fiche d'enseignement 4.1 Lausanne : Laboratoire de Gestion des Ecosystèmes, Ecole Polytechnique de Lausanne.

25. Krummel J.R., Gardner R.H., Sugihara G., O'neill R.V. \& Coleman P.R., 1987. Landscape patterns in a disturbed environment. Oikos, 48 (3), pp. 321-324.

26. Mandelbrot BB, 1994. The Fractal Geometry of Nature. New York: W. H. Freeman and Company.

27. Marage D., 2004. Déterminisme, dynamique et modélisation spatiale de la diversité floristique dans un contexte de déprise pastorale : Application à la gestion durable des espaces montagnards sous influence méditerranéenne. Thèse de doctorat, Ecole Nationale du Génie Rural, des Eaux et Forêts (ENGRE), Nancy, France, 236 p + annexes.

28. Mas JF, 2000. Une revue des méthodes et des techniques de télédétection du changement. Canadian Journal of Remote Sensing 26: 349- 62.

29. McGarigal K. \& Marks B.J., 1995. Fragstats: spatial pattern analysis program for quantifying structure. Department of Agriculture, Pacific Northwest Research Station General Technical Report PNW-GTR351. Oregon, USA, $132 \mathrm{p}$. 
30. ONU (Organisation des Nations-Unies), 2005. Objectifs du Millénaire pour le développement. New York, 48 p.

31. Orekan V. O.A, 2007. Implementation of the local land-use and landcover change model CLUE-s for Central Benin by using socioeconomic and remote sensing data. Ph.D. Thesis, Bonn University, 204 p.

32. Orthmann B., 2005. Vegetation ecology of a woodland-savanna mosaic in central Benin (West Africa): Ecosystem analysis with a focus on the impact of selective logging. PhD Thesis, Rostock University, Allemagne, $137 \mathrm{p}$.

33. PAGEFCOM, 2012. Schéma Directeur d'Aménagement Communal de Zagnanado, Ministère de l'Environnement, de l'Habitat et de l'Urbanisme ; $100 \mathrm{p}$.

34. Plagbeto A. H., 2014. Système d'Information Géographique (SIG) et télédétection dans l'étude de la dynamique de l'occupation du sol dans le bassin inférieur de l'Ouémé Bonou. DEA, Université d'AbomeyCalavi, 81p.

35. Sabatier R., Doyen L., Tichit M., 2010. Reconciling production and conservation in agrolandscapes: does landscape heterogeneity help? Innovation and Sustainable Development in Agriculture and Food (ISDA). Montpellier, France, 10 p.

36. Schlaepfer R, 2002. Analyse de la dynamique du paysage. Fiche d'enseignement 4.2. Lausanne : Laboratoire de Gestion des Ecosyst_emes, Ecole Polytechnique de Lausanne.

37. Song C, Woodcock CE, Seto KC, Lenney MP, Macomber SA, 2001. Classification and change detection using Landsat TM data : when and how to correct atmospheric effects? Remote Sensing and Environnement 75: 230-44.

38. Soumagbo., 2010. Etat de fragmentation du paysage dans la commune d'Abomey - Calavi. Mémoire de DESS, Université d'Abomey Calavi, $58 \mathrm{p}$.

39. Sounon Bouko B., 2011. Colonisation agricole et dégradation du couvert végétal dans le secteur Wari-Maro-Igbomakro au Bénin. Thèse de Doctorat, Université d'Abomey-Calavi, Bénin, 221 p.

40. Takou W. P., Boukpessi T., Djangbedja M. et Mama A., 2012. Apport de la télédétetion et des systèmes d'information géographiques dans l'étude de la dynamique des paysages végétaux de l'Ouest de la région des plateaux au Togo. Rev. Sc. Univ. Lomé (Togo), 009 : 29-48.

41. Tchamié T.T.K., 2002. Impacts des reboisements sur la flore du Togo. J. Rech.Sci. Univ. Lomé (Togo), 6 (2): 51 - 63.

42. Toko Mouhamadou I., 2014. Facteurs déterminants de la fragmentation des écosystèmes forestiers : cas des îlots de forêts 
denses sèches de la forêt classée des Monts Kouffé et de sa périphérie au Bénin, Thèse de Doctorat Unique, Université d'Abomey-Calavi, Bénin $231 \mathrm{p}$.

43. UNEP (United Nations Environment Programme) 2008. Africa: Atlas of Our Changing Environment. Division of Early Warning and Assessment (DEWA), UNEP, 393 p.

44. Wokou G., 2009. Agriculture et environnement sur le plateau d'Agonlin: systèmes culturaux et mutations environnementales, mémoire de DEA, UAC. 92 p. 